\title{
Non-extensive analysis of the seismic activity involving the 2011 volcanic eruption in El Hierro
}

\author{
F. J. Esquivel ${ }^{1, *}$ and J. M. Angulo ${ }^{1}$
}

${ }^{1}$ Department of Statistics and Operations Research, University of Granada, Campus de Fuente Nueva, E-18071 Granada,Spain; jesquivel@ugr.es, jmangulo@ugr.es

* Corresponding author

Abstract. This talk is focused on the assessment of the spatio-temporal dynamics and the scaling characteristics of the seismic activity associated with the volcanic eruption occurred during 2011 in El Hierro (Canary Islands, Spain). We use tools derived from the concepts of non-extensive statistical physics and multifractality.

Keywords. Entropy; Gutenberg-Richter power law; Multifractality; Non-extensivity; Seismicity.

\section{Introduction}

Empirical laws are widely used in the description of seismic phenomena, especially the GutenbergRichter law which, however, presents difficulties for events having small and high magnitudes. SotolongoCosta and Posadas (2004) propose a new frequency-magnitude distribution (FMD) model derived from fragment-asperity interaction and the non-extensive statistical physics. This model was revised by Silva et al. (2006), and later refined by Telesca (2011) in the form

$$
\log \left(\frac{N\left(m>m_{t h}\right)}{N}\right)=\left(\frac{2-q}{1-q}\right) \log \left\{1-\left(\frac{1-q}{2-q}\right)\left(\frac{10^{m_{t h}}}{a^{2 / 3}}\right)\right\}
$$

(see also Vallianatos et al. 2013). The constant $a$ represents the proportionality between the released energy and the fragments size, and $q$ is the non-extensivity parameter. Deviation of $q$ from 1 is interpreted as departure from equilibrium in the system due to strong spatial interactions; in particular, more events of relative larger magnitude can be expected as $q$ increases (e.g. Telesca 2011). Here, we adopt this model for fitting to observed data considering a weighting function based on the relation between magnitude and energy, $\phi(m)=\left(e^{m}\right)^{3 / 2}$.

On the other hand, earthquakes are characterized by certain forms of scaling behaviour intrinsic to spatio-temporal dynamics (see, for example, Bak et al. 1987). State distributions associated with such 
systems are formally described in terms of multifractal measures. Angulo and Esquivel (2014) formulate a normalized coefficient useful to quantify the degree of association between marginal components, in the generalized dimensions domain. We propose here a non-extensive form of this coefficient in terms of the generalized dimensions based on Tsallis entropy also introduced in the former work.

In this work, we assess possible relational elements of the submarine volcanic eruption occurred during 2011 in El Hierro island with both the spatio-temporal evolutionary behaviour and the scaling properties of seismic activity before, during and after it. The geophysics of the island has been widely studied and it is known that there are three volcanic rifts around it (Acosta et al. 2005). In particular, we analyze the epicenter coordinates, time and magnitude components of the earthquake catalogue between 19 July 2011 and 7 January 2012. The main singularities of the series are the large number of events occurred (more than 11.000 events in just 173 days) and the fact that a volcanic eruption is involved. According to Ibáñez et al. (2012), the series can be divided into three well-separated spatio-temporal clusters.

In the next section, we study the spatio-temporal structural characteristics and scaling properties of the earthquake swarm. In the concluding section, we summarize the main results and related interpretations in connection with the aims proposed.

\section{Spatio-temporal analysis of El Hierro volcanic seismicity}

\subsection{FMD}

We perform three analyses to assess the structural dynamics of the series by means of the FMD: firstly, we study the temporal evolution considering data segments with fixed a number of events (which is chosen large enough to ensure a good fitting but not too large in order to detect local changes in the behaviour); secondly, we carry out a spatial analysis evaluating the local variations of the estimated parameter values; finally, we complete the study with the spatio-temporal analysis combining both methodologies.

Temporal analysis The temporal dynamics of the $a$ and $q$ parameters is shown in Figure 1. The variations of $a$ are studied in reference to the three phases (referred as A, B and C) mentioned before. In addition, we can observe that during the days previous to the volcanic eruption great amounts of energy are released in proportion to the fragments size. As for parameter $q$, larger values occur during the volcanic eruption, indicating that the physical state is farther from equilibrium.
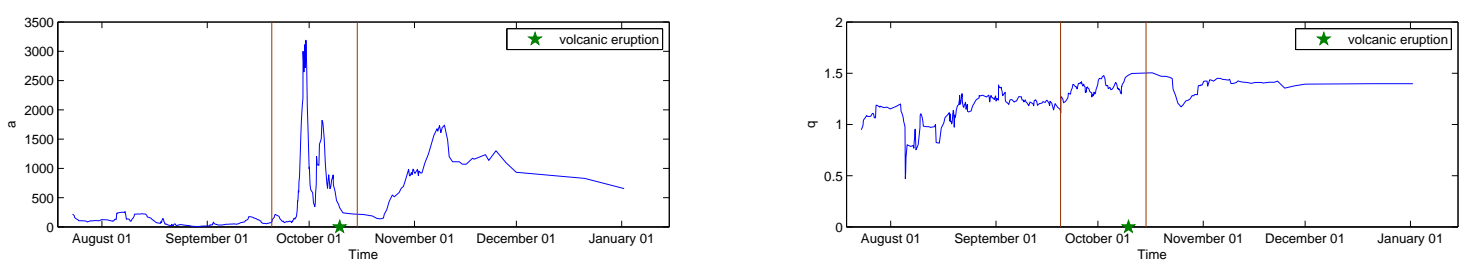

Figure 1: Estimated values of parameters $a$ and $q$ over time based on sliding windows (size 300 events) considering an overlapping of 90 per cent.

Spatial analysis We study the spatial dynamics of the series considering the whole period and the three phases A, B and C. The variations in the estimated values of parameters $a$ and $q$, depicted in Figure 2, indicate that, in both cases, the larger values are highly concentrated close to the South rift. In fact, it is clearly visible that these values are mainly due to events occurring in Phase B, when the volcanic 
eruption began to be registered. Furthermore, for both parameters it is important to highlight that the most significant changes in the spatial location are associated with the latitude coordinate.
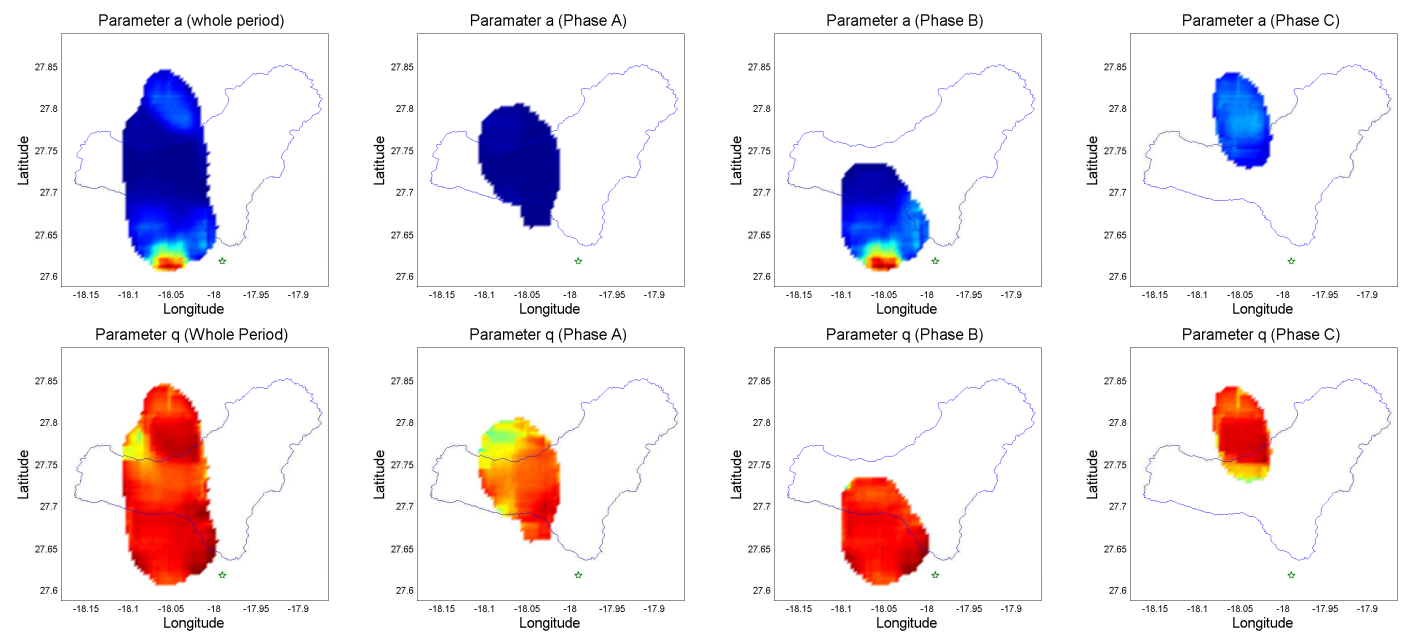

Figure 2: Distribution of the variation of $a$ and $q$ for the whole period and the three phases A, B and C.

Spatio-temporal analysis Figure 3 shows the variations in the spatial location (longitude and latitude separately) of the maximum value of the each parameter, $a$ and $q$, for data segments based on temporal sliding windows of size 20 days (the last two months are excluded in this analysis due to the low density of events). We can see how, as the volcanic eruption is approaching, the maximum values obtained for latitude begin to move towards the eruption location, especially in the case of parameter $a$. Regarding the longitude there are not significant variations, in correspondence with previous results.
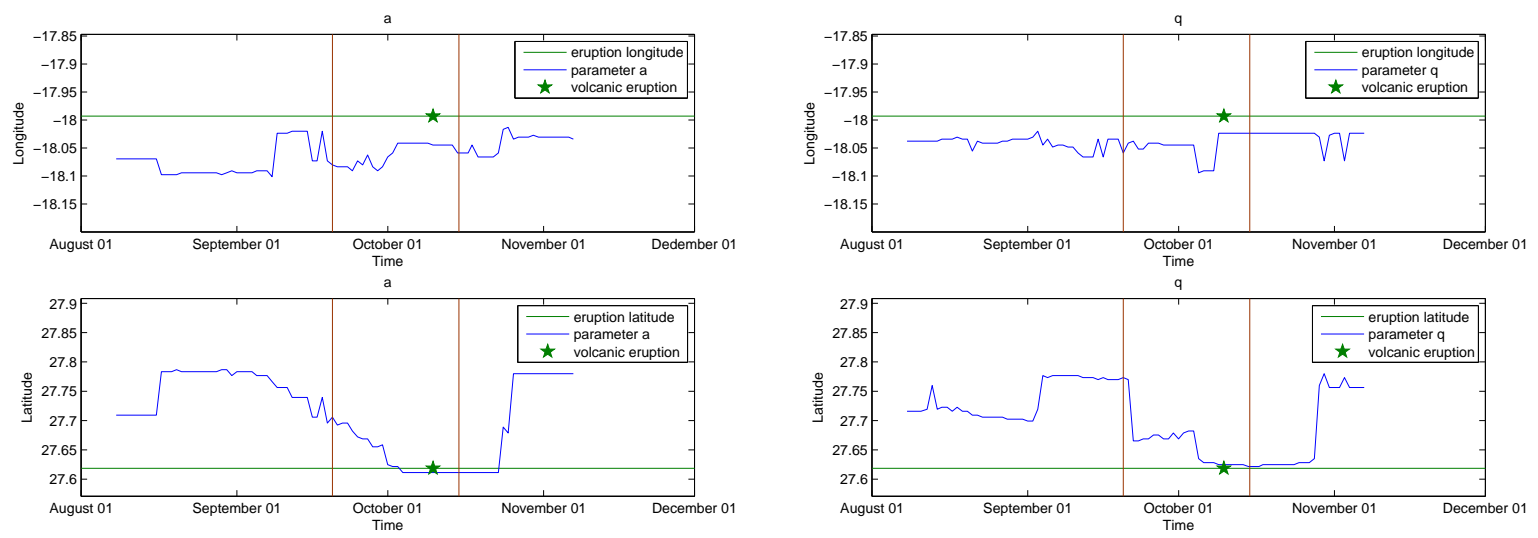

Figure 3: Temporal evolution of the location, for longitude (top) and latitude (bottom), associated with the maximum estimated value of the parameters $a$ (left) and $q$ (right).

\subsection{Dependence analysis}

We complete the study with the assessment of the dependence between the locations of the events and their magnitudes. Figure 4 indicates that, in the interaction $(X, Y) \leftrightarrow M$, relevant changes are observed during the days previous to the volcanic eruption. In particular, large fluctuations are related to the interaction $Y \leftrightarrow M$, whereas the visible increasing trend is related to the interaction $X \leftrightarrow M$, also with a significant degree of association between spatial coordinates. 

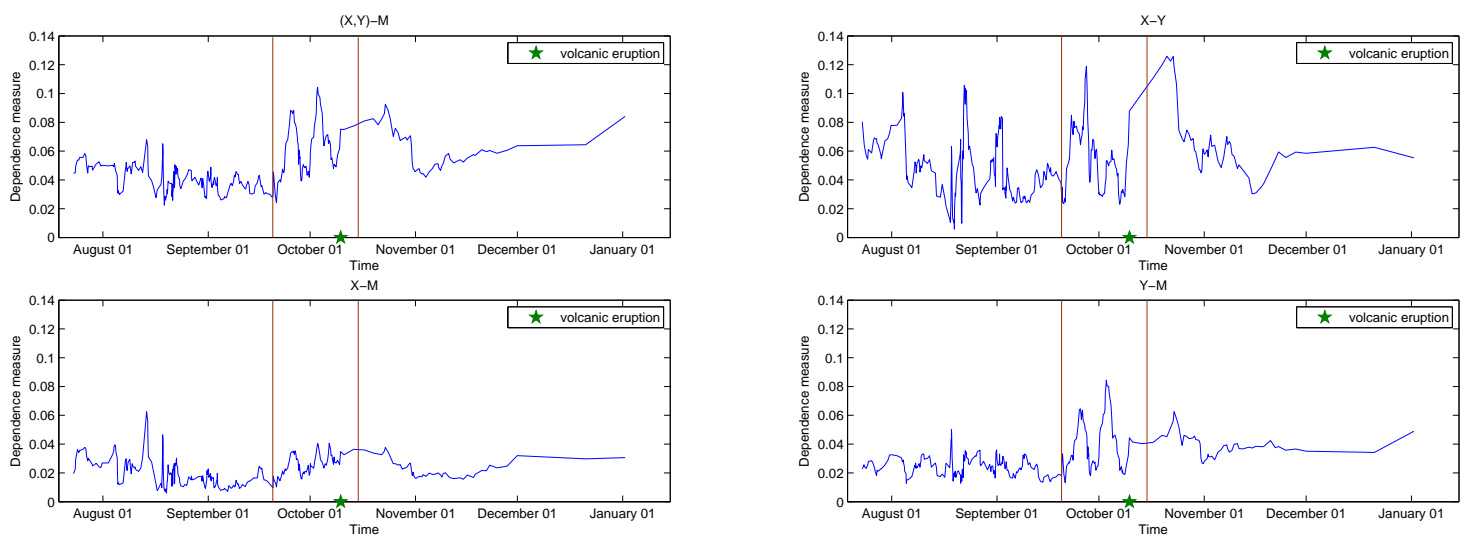

Figure 4: Distribution of the variation of the dependence coefficient over time based on sliding windows (size 20 days), considering an overlapping of 90 per cent, for the different dimensional interactions.

\section{Conclusion}

The study shows significant changes previous to the volcanic eruption in the spatio-temporal dynamics, based on the assessment of structural characteristics and scaling properties of the seismic activity. The combination of these techniques can be seen as a suitable tool in the continuous monitoring of volcanic activity.

Acknowledgments. This work has been partially supported by Spanish grant MTM2012-32666 (cofinanced by FEDER).

\section{References}

[1] Acosta, J., Uchupi, E., Smith, D., Muñoz, A., Herranz, P., Palomo, C., Llanes, P., Ballesteros, M. (2005). Comparison of volcanic rifts on La Palma and El Hierro, Canary Islands and the Island of Hawaii. Marine Geophysical Researches 24, 59-90.

[2] Angulo, J. M., Esquivel, F. J. (2014). Structural complexity in space-time seismic event data. Stochastic Environmental Research and Risk Assessment 28, 1187-1206.

[3] Bak, P., Tang, C., Weisenfeld, K. (1987). Self-organized criticality: An explanation of 1/f noise. Physical Review Letters 59, 381-384.

[4] Ibáñez, J. M., De Angelis, S., Díaz-Moreno, A., Hernández, P., Alguacil, G., Posadas, A., Pérez, N. (2012). Insights into the 2011-2012 submarine eruption off the coast of El Hierro (Canary Islands, Spain) from statistical analyses of earthquake activity. Geophysical Journal International 191, 659-670.

[5] Silva, R., França, G. S., Vilar, C. S., Alcaniz, J. S. (2006). Nonextensive models for earthquakes. Physical Review E 73, 026102.

[6] Sotolongo-Costa, O., Posadas, A. (2004). Fragment-asperity interaction model for earthquakes. Physical Review Letters 92, 048501.

[7] Telesca, L. (2011). Tsallis-based nonextensive analysis of the Southern California seismicity. Entropy 13, 1267-1280.

[8] Vallianatos, F., Michas, G., Papadakis, G., Tzanis, A. (2013). Evidence of non-extensivity in the seismicity observed during the 2011-2012 unrest at the Santorini volcanic complex, Greece. Natural Hazards and Earth System Sciences 13, 177-185. 